\title{
Chaos and nonlinear dynamics: Advances and perspectives
}

\author{
G. Károlyi ${ }^{1,2}$, A. de Moura ${ }^{3}$, M.C. Romano ${ }^{3}$, M. Thiel ${ }^{3}$, and J. Kurths ${ }^{4,5,6}$ \\ 1 Centre for Applied Dynamics Research, King's College, University of Aberdeen, \\ AB24 3UE Aberdeen, Scotland, UK \\ 2 Department of Structural Mechanics, Budapest University of Technology and Economics, \\ 1521 Budapest, Hungary \\ 3 Department of Physics, King's College, University of Aberdeen, AB24 3UE Aberdeen, Scotland, UK \\ 4 Center for Dynamics of Complex Systems, University of Potsdam, 14415 Potsdam, Germany \\ ${ }^{5}$ Institute of Physics, Humboldt University Berlin, 12489 Berlin, Germany \\ 6 Potsdam Institute for Climate Impact Research, 14412 Potsdam, Germany
}

Chaotic behaviour of nonlinear systems has been one of the most fruitful additions to physics and applied mathematics in the latter part of the twentieth century. It has evolved into a mature discipline, which keeps researchers active in many areas of science. This special issue of the European Physical Journal is dedicated to reporting the latest advances in that area. The papers collected here span the full spectrum of the field, and show that it continues to be an exciting and active area of research. We have grouped all papers into four sections: a theoretical section, a section about synchronisation, a section on fluid dynamics, and a section on applications. We will now briefly address each paper and outline the most important results.

Despite the fact that Dynamical Systems is a mature area of science, its fundaments and theoretical basis are still a source of many fascinating problems. One of the most exciting areas of dynamical systems theory is high-dimensional Hamiltonian dynamics. An important problem in the numerical study of Hamiltonian systems of high dimension is to efficiently compute the slow diffusion of trajectories near quasiperiodic tori. The first paper in this collection addresses this issue, and proposes an efficient method to calculate the $\mathrm{GALI}_{k}$ coefficients, based on the singular value decomposition algorithm; the authors use their method to understand diffusion and other issues in the Fermi-Pasta-Ulam system [1].

Another crucial area within the theory of dynamical systems deals with the development of time-series analysis techniques. Since Takens' theorem, embedding and time-series analysis have been the corner stones of many applications of dynamical systems theory. These topics also have a rich theoretical background, which is a research topic of its own. Vaidya and Majumder [2] investigate the cause of spurious instabilities often found in model equations reconstructed from time series data; they find that this is related to a topological foliation caused by the embedding. Henschel et al. [3] develop a multi-variate analysis method to adapt time-series analysis techniques for point processes - that is, data consisting of only a set of time points where a certain event happened, such as the firing of a neuron; this is crucial for the analysis of biomedical data. Kwasniok [4] tackles yet another aspect of time-series analysis: predictability. By fitting a hidden Markov chain model to time series data, he is able to predict the probabilities of visiting specific regions in phase space; the method is validated with the forced Lorenz system.

Another major area in the theory of dynamical systems is bifurcation analysis. Non-smooth dynamical systems are well known for the rich set of bifurcations they display. Taralova [5] investigates a new bifurcation found in a particular class of piece-wise linear maps; this bifurcation leads to the creation of a chaotic orbit. A different kind of non-smoothness is studied by Pokorny and Klic in their paper [6], wherein they investigate the dynamics of a vector field which alternates periodically in time between two different forms. They show that the period 
map can be generated by a single, autonomous vector field, generalizing a result which was previously known only for linear systems. Nagaraj et al. [7] study how the period of numerically simulated chaotic maps, which are finite because of the limited accuracy of floating-point calculations, is affected by on-off switching of chaotic motion. They find that the average period increases for random switching of chaos, and use this to implement a chaotic random number generator.

Another important aspect of the theory of dynamical systems is the very strong connections with statistical physics. The statistical properties of fluctuations of chaotic systems are best characterized by statistical structure functions. Kobayashi [8] propose a method to calculate the large deviation statistical characteristic functions for chaotic dynamical systems, and illustrate by applying it to simple systems.

The final article of the theoretical part of this collection of papers revisits the problem of scars in quantum chaos. The traditional way to understand why some periodic orbits have a large effect on the wave function of the equivalent quantum system is through the constructive interference generated by the periodic recurrences of a wave-packet along the periodic orbit. Borondo et al. [9] look more deeply into this phenomenon, and take into account other, higherorder recurrences, and show that they can have important effects on quantum transport.

A collection of cutting-edge research papers in nonlinear dynamics and chaos theory would hardly be complete without some titbits on the latest results on synchronisation. Synchronisation is not only ubiquitous in nature, leading to most spectacular phenomena like the perfectly timed flashing of swarms of male fireflies in South-East Asia, but it also is at the heart of our thinking itself: without the synchronised firing of billions of neurons, none of our thoughts would form. Most naturally, researchers are intrigued by these captivating processes.

The first paper in this section on synchronisation is entitled "A linear path toward synchronisation" [10]. Roberts and Teodorescu study how synchronisation comes about in large ensembles of oscillators. They find a transition to a simultaneous behaviour of the population, which is different from Kuramoto's standard model. The results are obtained from a mathematical model, which incorporates the topology of the network, i.e., which oscillator is linked to which other, and the coupling itself, i.e., what "force" interacts between the oscillators. Both the topology and the coupling are crucial for the synchronisability of the network. Blakely and Corron exploit different coupling schemes and synchronisation to time shift chaotic signals [11]. This method can be used to direct the output of beam forming array antennas. Directed transfer of information mediated by synchronisation is one of the aspects that Baptista et al. investigate [12]. They find that more pronounced synchronisation makes information transfer more effective in certain circumstances. This result indicates how, e.g., the brain might use synchronisation to process its sensorial input. Espositi and Signorini show how the connectivity of a network is generated [13]. During the brain's development networks are formed and pruned. The coupling determines how/if the brain functions later in life. The authors successfully apply a mathematical approach to model parts of the mammalian brain. The last paper in this section moves away from the brain and deals with synchronisation of chemical systems. Taylor et al. observe phase clustering in globally coupled Belousov Zhabotinsky oscillators [14]. All of these papers have obvious and important applications. However, due to the overarching topic, they form the chapter on synchronisation.

In the section on fluid dynamics, the authors treat the motion of particles transported by fluid flows. From the simplest case, passive advection, where the particles passively follow the velocity field of the fluid, to the interplay of fluid motion and chemical activity, it is a very important subfield of nonlinear dynamics. Chaos in fluid flows has long been attracting the interest because the patterns usually hidden in an abstract phase space are visible to the naked eye of researchers.

The paper by Ravi et al. [15] sheds light on the very complex behaviour of vortex shedding in the wake of two obstacles at a close distance apart. With an accurate numerical technique they are able to follow the intricate structure of the flow patterns generated by the viscous fluid, and to give a statistical analysis of the fluid-obstacle interaction.

Not only the fluid motion itself, but also the chemical activity superimposed on fluid flows can lead to very interesting patterns. The experimental investigations by Tinsley et al. [16] reveal stabilized waves in the Belousov-Zhabotinsky reaction that can be directionally controlled 
by intensity gradients in the applied illumination. Also in the Belousov-Zhabotinsky reaction, Fernández-García and Pérez-Muñuzuri [17] find a superdiffusive front propagation of Faraday waves. In their experimental studies the chemically active fluid, forced by the Faraday instability, exhibited Lévy-flights, where the wave fronts followed an alternating sequence of sticking and excursion periods.

There is a vast number of applications of the theory of nonlinear dynamics to a large variety of real-world systems. This broad range of applications is reflected in the last section of this special issue, which deals with topics ranging from the dynamics of biological molecules, such as DNA, to the control of chaos in satellite power supply subsystems.

In the first paper of this section, the mechanics of topoisomerase, a specialised enzyme that decreases the stress in the strongly twisted DNA molecule, is examined by Thompson [18]. The dynamical processes involved generate many different outcomes, including co-existing stable equilibrium states. Losada et al. [19] apply the theoretical results from chaos in Hamiltonian systems to the vibrations of the LiCN molecule. It is shown that by using frequency maps, a realistic potential for the vibrations of the molecule is described accurately. Portela et al. [20] proposes the use of simple two-dimensional, area-preserving maps to approximate the magnetic field line equations that describe the magnetic field in a tokamak. This approach is shown to be advantageous compared to the direct numerical integration of the field line equations, since the latter is a lengthy procedure and requires a very time-consuming analysis.

Moroz [21] identifies the underlying chaotic attractor in a nonlinear three-dimensional model for a self-exciting Faraday disk dynamo. Using unstable periodic orbits the author characterises the template for the chaotic attractor and analyses how this template is influenced by changes in the bifurcation parameter. Macau et al. [22] apply chaos control techniques to increase the region that can be efficiently used to supply the power requests of an artificial satellite. The power supply system is the most critical system in any spacecraft. Therefore, it is crucial to develop techniques to extend the applicability region of the satellite power system. This can be achieved in highly nonlinear regions of the parameter space by applying chaos control techniques. In the last paper of this section, Sitnikova et al. [23] model and analyse the dynamic behaviour of an impact oscillator with a shape memory alloy (SMA) constraint. Shape memory alloys are metallic alloys which can be transformed to a different solid phase by temperature variations. Due to the mechanical properties of the SMAs and to the non-smooth nature of the impacts, the authors have identified five different modes of operation. Futhermore, they show that the control of the complex dynamical responses of this system can be used to reduce vibrations.

Hence, the power of nonlinear dynamics to describe and analyse such a range of different real-world sytems is demonstrated in this section, which also gives an indication of future perspectives.

In this special issue we outline some of the most active research fields in nonlinear dynamics. We hope to convince the reader that the field is not only very vivid but also highly interesting. In an obituary to Edward Lorenz, one of the founders of chaos theory, it was argued that nonlinear dynamics was one of the major revolutions in $20^{\text {th }}$ century science (next to relativity and quantum theory). It is certainly remarkable how the ideas of nonlinear dynamics and chaos theory pervade practical all natural sciences. Nonlinear dynamics is therefore a very interdisciplinary science, and attracts scientists from all over the world. The articles we present in this issue are based on presentations given at the conference "Chaos and Nonlinear Dynamics: Advances and Perspectives". This conference was held in 2007 at the University of Aberdeen on the ocassion of the $60^{\text {th }}$ birthday of one of the most prolific scientists in this field, Prof. Celso Grebogi, whom we want to thank for his stimulating and exciting contributions to our field of research.

G. Károlyi, A.P.S. de Moura, M.C. Romano, M. Thiel, J. Kurths Guest Editors 


\section{References}

1. C. Skokos, et al., Eur. Phys. J. Special Topics 165, 5 (2008)

2. P.G. Vaidya, S. Majumder, Eur. Phys. J. Special Topics 165, 15 (2008)

3. K. Henschel, et al., Eur. Phys. J. Special Topics 165, 25 (2008)

4. F. Kwasniok, Eur. Phys. J. Special Topics 165, 35 (2008)

5. I. Taralova, Eur. Phys. J. Special Topics 165, 45 (2008)

6. P. Pokorny, A. Klic, Eur. Phys. J. Special Topics 165, 61 (2008)

7. N. Nagaraj, et al., Eur. Phys. J. Special Topics 165, 73 (2008)

8. M.U. Kobayashi, Eur. Phys. J. Special Topics 165, 85 (2008)

9. F. Borondo, et al., Eur. Phys. J. Special Topics 165, 93 (2008)

10. D.C. Roberts, R. Teodorescu, Eur. Phys. J. Special Topics 165, 103 (2008)

11. J.N. Blakely, N.J. Corron, Eur. Phys. J. Special Topics 165, 111 (2008)

12. M.S. Baptista, et al., Eur. Phys. J. Special Topics 165, 119 (2008)

13. F. Esposti, M.G. Signorini, Eur. Phys. J. Special Topics 165, 129 (2008)

14. A.F. Taylor, et al., Eur. Phys. J. Special Topics 165, 137 (2008)

15. A.B. Ravi, et al., Eur. Phys. J. Special Topics 165, 151 (2008)

16. M.R. Tinsley, et al., Eur. Phys. J. Special Topics 165, 161 (2008)

17. G. Fernández-García, V. Pérez-Muñuzuri, Eur. Phys. J. Special Topics 165, 169 (2008)

18. J.M.T. Thompson, Eur. Phys. J. Special Topics 165, 175 (2008)

19. J.C. Losada, et al., Eur. Phys. J. Special Topics 165, 183 (2008)

20. J.S.E. Portela, et al., Eur. Phys. J. Special Topics 165, 195 (2008)

21. I.M. Moroz, Eur. Phys. J. Special Topics 165, 211 (2008)

22. E.E.N. Macau, et al., Eur. Phys. J. Special Topics 165, 221 (2008)

23. E. Sitnikova, et al., Eur. Phys. J. Special Topics 165, 229 (2008) 